\title{
Spectral entropy for assessing the depth of propofol sedation
}

\author{
Mi-Young Kwon ${ }^{1}$, Seung-Yun Lee ${ }^{2}$, Tae-Yop Kim³ , Duk Kyung Kim³ ${ }^{3}$ Kyoung-Min Lee ${ }^{3}$, Nam-Sik Woo ${ }^{3}$, \\ Young-Jae Chang ${ }^{1}$, and Myung Ae Lee ${ }^{1}$ \\ Department of Anesthesiology, ${ }^{1}$ National Medical Center, Seoul, ${ }^{2}$ School of Medicine, Jeju National University, Jeju, \\ ${ }^{3}$ Konkuk University Medical Center, Research Institute of Biomedical Science, Konkuk University School of Medicine, Seoul, Korea
}

Background: For patients in the intensive care unit (ICU) or under monitored anesthetic care (MAC), the precise monitoring of sedation depth facilitates the optimization of dosage and prevents adverse complications from underor over-sedation. For this purpose, conventional subjective sedation scales, such as the Observer's Assessment of Alertness/Sedation (OAA/S) or the Ramsay scale, have been widely utilized. Current procedures frequently disturb the patient's comfort and compromise the already well-established sedation. Therefore, reliable objective sedation scales that do not cause disturbances would be beneficial. We aimed to determine whether spectral entropy can be used as a sedation monitor as well as determine its ability to discriminate all levels of propofol-induced sedation during gradual increments of propofol dosage.

Methods: In 25 healthy volunteers undergoing general anesthesia, the values of response entropy (RE) and state entropy (SE) corresponding to each OAA/S (5 to 1) were determined. The scores were then analyzed during each 0.5 $\mathrm{mcg} / \mathrm{ml}$ - incremental increase of a propofol dose.

Results: We observed a reduction of both RE and SE values that correlated with the OAA/S (correlation coefficient of 0.819 in RE-OAA/S and 0.753 in SE-OAA/S). The RE and SE values corresponding to awake (OAA/S score 5), light sedation (OAA/S 3-4) and deep sedation (OAA/S 1-2) displayed differences $(\mathrm{P}<0.05)$.

Conclusions: The results indicate that spectral entropy can be utilized as a reliable objective monitor to determine the depth of propofol-induced sedation. (Korean J Anesthesiol 2012; 62: 234-239)

Key Words: Entropy, Propofol, Sedation.

Received: April 12, 2011. Revised: 1st, May 30, 2011; 2nd, June 21, 2011; 3rd, July 1, 2011; 4th, July 12, 2011. Accepted: July 15, 2011.

Corresponding author: Tae-Yop Kim, M.D., Department of Anesthesiology, Konkuk University Medical Center, Research Institute of Biomedical Science, Konkuk University School of Medicine, 4-12, Hwayang-dong, Gwangjin-gu, Seoul 143-729, Korea. Tel: 82-2-2030-5445, Fax: 82-2-20305449, E-mail: taeyop@kuh.ac.kr

(ㄷ) This is an open-access article distributed under the terms of the Creative Commons Attribution Non-Commercial License (http:// creativecommons.org/licenses/by-nc/3.0/), which permits unrestricted non-commercial use, distribution, and reproduction in any medium, provided the original work is properly cited. 


\section{Introduction}

The precise monitoring of sedation depth facilitates the optimization of dosage and prevents adverse complications from under- or over-sedation. For this purpose, conventional subjective sedation scales, such as the OAA/S or the Ramsay scale, have been widely used for the management of patients in the intensive care unit (ICU), under monitored anesthetic care (MAC) or surgical patients in regional anesthesia. However, when these procedures are performed, they frequently disturb the patient's comfort and compromise the already wellestablished sedation. Thus, the ability to apply other reliable objective sedation scales that do not cause disturbances would be beneficial in the management of sedated patients. This concept raises concerns regarding the possibility of adopting EEG-based monitoring, a method applied to guarantee sufficient intraoperative anesthesia depth, as an objective sedation scale to replace conventional subjective sedation scales.

Previous investigations have analyzed the relationship between EEG-based monitors, such as the bispectral index (BIS) or spectral entropy (SE) and the conventional sedation scales in sedated patients. Despite a favorable correlation between the EEG-derived monitor and the conventional sedation scale, these studies did not indicate the monitor's ability to discriminate all levels of sedation depth, as defined by the conventional sedation scales. Furthermore, over-sedation during therapy guided by the BIS monitor in addition to an insufficient correlation between the depth of sedation determined by the BIS value and hypnotic dosage were reported $[1,2]$.

There are several reasons behind the insufficiency of the EEG-derived monitor to discriminate between all depths of sedation in order to guide sedation therapy [3-5]. First, the electromyographic (EMG) activity from eye movements can compromise the monitor's ability, particularly in lightly sedated patients who are not paralyzed [4]. The entropy monitoring consists of SE, devoid of EMG interference, and may thus be beneficial in this situation. Second, when the combined use of opioids and hypnotics is applied, the overall depth of sedation is synergistic via the extra-cortical pathway. However, the EEG-derived monitor (which reflects cortical activity in the determination of sedation depth) cannot detect extracortically potentiated sedation; indeed, even the BIS value that defines sufficient anesthesia depth may shift to higher values in the combined use of opioids and hypnotics $[2,3,5,6]$. This discrepancy would be exaggerated during opioid-accentuated sedation therapy in the ICU.

For establishing the efficacy of the EEG-derived monitor (for both the titration of hypnotic doses and guiding the depth of hypnotic-based sedation), the current study analyzed the changes of entropy corresponding to the OAA/S value during a progressive deepening of propofol-induced sedation except for the opioids due to the undetectable sedative effect.

\section{Materials and Methods}

The study was approved by the Institutional Review Board and written informed consent from the 25 patients $(20-54$ years of age), undergoing elective orthopedic surgery with general anesthesia, were enrolled in this prospective study. Exclusion criteria were a history of cardiac, pulmonary, liver or renal disease, or significant obesity. Long term user of central nervous system activator drugs, including benzodiazepines or opiates was excluded. An intravenous line was placed in the patient's forearm and glycopyrrolate $(0.2 \mathrm{mg})$ was administered intravenously in the patient holding area. Upon the patient's arrival to the operating room, routine monitoring including an electrocardiogram, pulse oximetry and non-invasive blood pressure were initiated. To monitor spectral entropy, a threeelement electrode (Entropy sensor ${ }^{\mathrm{TM}}$ Datex-Ohmeda, Finland) with a plug-in monitor (M-Entropy plug-in module, DatexOhmeda, Finland) was applied, as outlined in the manufacturer's manual.

Patients were instructed to close and open their eyes when the investigator called their name and shook their body to determine the OAA/S score (from 5 to 1 ) during the study period (Table 1). After the patient became comfortable in the operating room, baseline values for the RE (100 to 0), SE (91 to 0) were recorded and compared to the $\mathrm{RE}$ and $\mathrm{SE}$ values corresponding to the OAA/S score of 5 .

Propofol was administered using a computer-assisted, targetcontrolled infusion (TCI) device (Orchestra ${ }^{\mathrm{TM}}$ and Base $\mathrm{A}^{\mathrm{TM}}$, Fresenius Vial Infusion Systems, France) with a maximum flow rate of $1,200 \mathrm{ml} / \mathrm{h}$, according to the pharmacokinetic-dynamic model published by Schnider and coworkers $[7,8]$. Initially, the target effect-site concentration of propofol was set to $1.0 \mu \mathrm{g} /$ $\mathrm{ml}$ and increased incrementally by $0.5 \mu \mathrm{g} / \mathrm{ml}$ to deepen the sedation depth. At each increment, the $\mathrm{RE}$ and $\mathrm{SE}$ values were recorded and an equilibrium of propofol plasma and effect-site concentrations were displayed on the TCI device. OAA/S scores were then immediately determined. The following sequence was maintained throughout the study protocol: (1) RE and SE

Table 1. Observer's Assessment of Alertness/Sedation Scale

\begin{tabular}{cl}
\hline Score & Responsiveness \\
\hline 5 & Awake and responds to name, spoken in normal tone \\
4 & Lethargic response to name, spoken in normal tone \\
3 & Responds only after name called loudly and/or repeatedly \\
2 & Responds only after name called loudly and after mild \\
& shaking of body \\
1 & No response after name is called loudly with mild shaking \\
\hline
\end{tabular}


scores, (2) the OAA/S score and (3) the RE and SE values were defined as those corresponding to each OAA/S score.

During propofol administration a face mask was fitted to measure the $\mathrm{ET}_{\mathrm{CO}_{2}}$. Observing the slugged respiration, assisted or controlled ventilation of $\mathrm{O}_{2}(6 \mathrm{~L} / \mathrm{min})$ was applied to maintain the $\mathrm{ET}_{\mathrm{CO}_{2}}$ within 35-40 mmHg. After achieving the $\mathrm{OAA} / \mathrm{S} 1$, the study was completed and rocuronium (0.6-0.9 $\mathrm{mg} / \mathrm{kg}$ ) was administered to facilitate tracheal intubation.

The correlations between the RE and SE values and the OAA/S score were determined using linear regression and Spearman's correlation. To evaluate the ability of entropy to discriminate between the status of awake, light sedation and deep sedation, RE and SE corresponding to the OAA/S scores of $5,3-4$, and 1-2 were analyzed by using the Kruskal-Wallis one way analysis of variance, and pair-wise comparisons among the OAA/S groups were performed using a Dunn's test. The analyses were performed using the statistical program (Sigma Stat ${ }^{\mathrm{TM}}$ ver. 3.1, Systat Software, USA).

\section{Results}

One patient was excluded from the study due to a repetition of abrupt fluctuations in the RE and SE values on calling the patient's name and an intermittent agitated response. Another patient was excluded due to unexpected heavy sedation at lower doses of propofol resulting in failure to determine the status of the OAA/S score of $4-3$.

Data from twenty-five patients ( 12 male / 13 female) of ages (41 \pm 10 years), height ( $167 \pm 8 \mathrm{~cm}$ ) and weight ( $65 \pm 7 \mathrm{~kg}$ ) were included for this analysis. The median values of the RE and SE values corresponding to OAA/S scores of 5, 4, 3, 2 and 1 are shown in Table 2. The changes of RE and SE values showed significant correlation and predictability with changes in the OAA/S score $(\mathrm{n}=75$, correlation coefficients of 0.811 and 0.802 , respectively, and $r=0.811, r^{2}=0.657, y=17.987+15.653 x$; and $r=0.802, r^{2}=0.644, y=16.1+14.4 x$, respectively)(Fig. 1 ). The median $(25-75 \%)$ of the RE and SE values corresponding to awake, light sedation and deep sedation were significantly different (96 [94-98], 76 [55-93] and 40 [30-45], respectively; $\mathrm{P}<0.001)$ and (86 [85-89], 69 [50-81] and 36 [29-39], respectively; $\mathrm{P}<0.001)$, and their pair-wise comparisons showed significantly different values $(\mathrm{P}<0.001)$ (Fig. 2).

\section{Discussion}

The OAA/S score was selected for this study as it has been well regarded as demonstrating correlation with hypnoticbased sedation, even in the ICU setting, in previous prospective studies [9]. Moreover, its efficacy was equal to the Ramsay scale as determined by comparison with other EEG-based monitors,

Table 2. Response Entropy (RE) and State Entropy (SE) Values for Each OAA/S Score

\begin{tabular}{cccccc}
\hline & OAA/S 5 & OAA/S 4 & OAA/S 3 & OAA/S 2 & OAA/S 1 \\
\hline $\mathrm{RE}$ & $95.7(91-99)$ & $84.7(46-99)$ & $63.4(30-99)$ & $42.3(25-95)$ & $38.7(15-69)$ \\
$\mathrm{SE}$ & $86.5(82-90)$ & $73.7(40-87)$ & $57.5(32-88)$ & $39.1(24-89)$ & $33.8(14-60)$ \\
\hline
\end{tabular}

Data shown are median values (range; minimum-maximum). The median values corresponding to the OAA/S scores in both RE and SE groups displayed a significant difference $(\mathrm{P}<0.001)$. OAA/S: Score of Observer's Assessment of Alertness/Sedation.

A

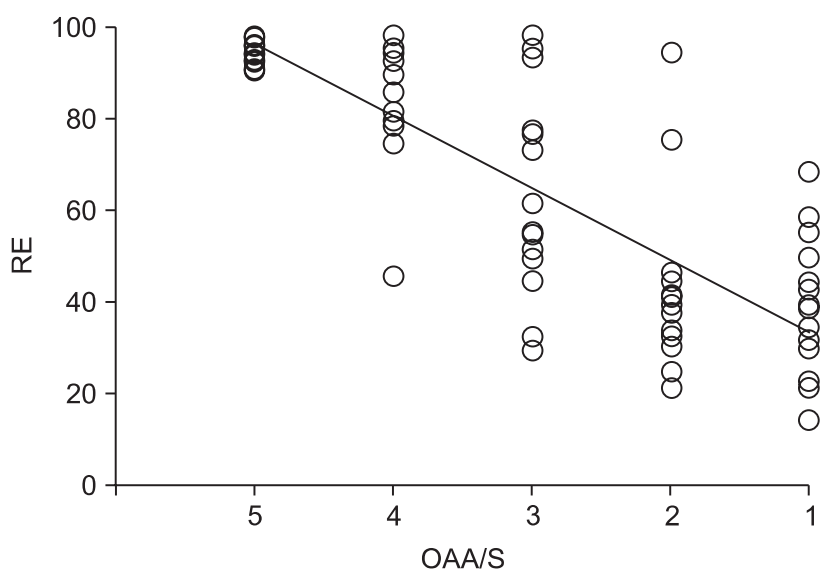

B

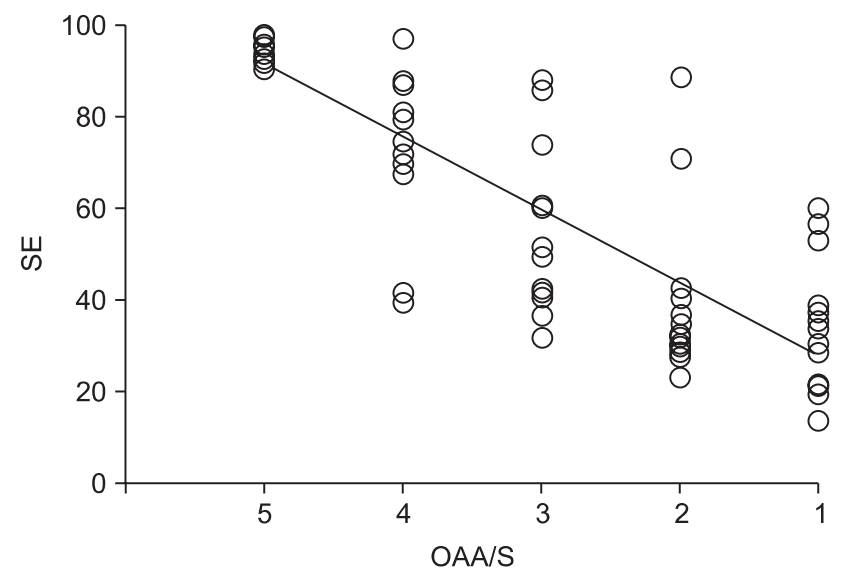

Fig. 1. Response entropy (A) and state entropy (B) values corresponding to each of the observer's assessment of alertness/sedation (oaa/s) score. The values of both RE and SE displayed a significant correlation with the OAA/S scores $\left(\mathrm{r}=0.811, \mathrm{r}^{2}=0.657, \mathrm{y}=17.987+15.653 \mathrm{x}\right)$ and $(\mathrm{r}=$ $\left.0.802, \mathrm{r}^{2}=0.644, \mathrm{y}=16.1+14.4 \mathrm{x}\right)$, respectively. 
A

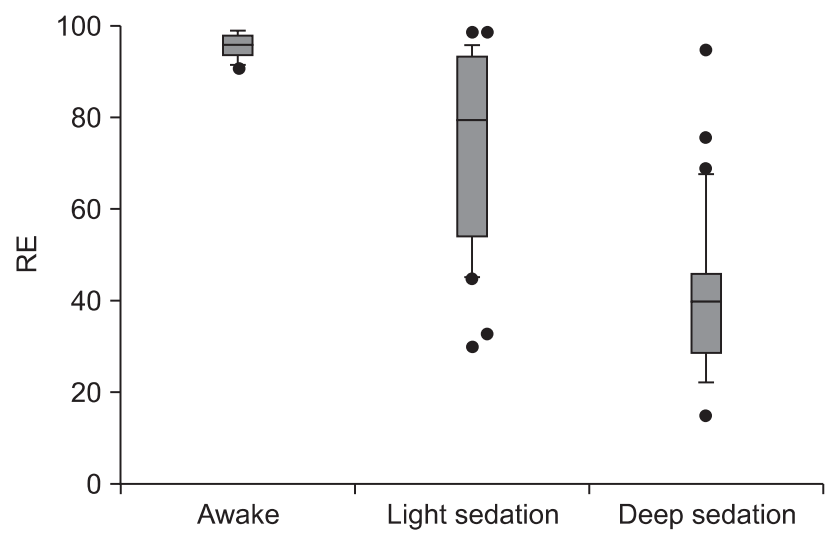

B

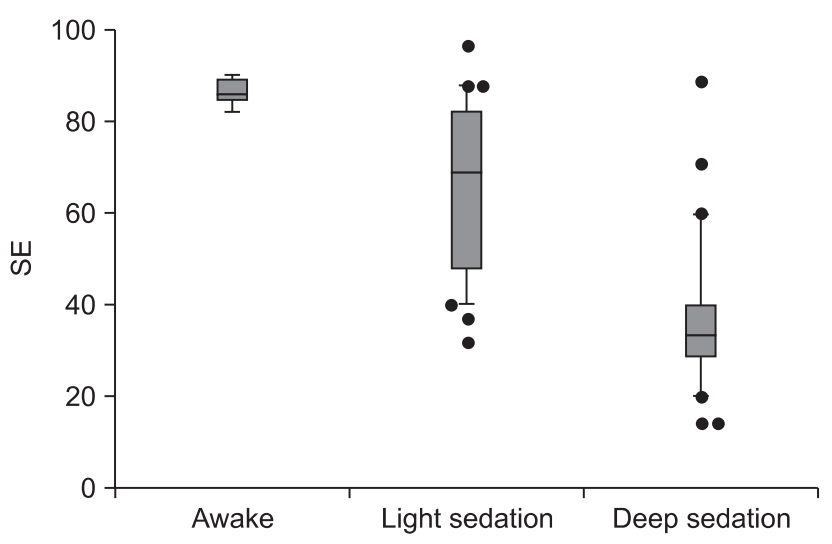

Fig. 2. Response entropy (A) and state entropy (B) during various sedation levels. Each sedation group differed from the others by all pair-wise multiple comparison procedures (Dunn's method $[\mathrm{P}<0.005]$ ).

although it has several limitations and variations regarding its clinical application [9-14].

The role of the EEG-derived monitor in the operating room is focused on ensuring a sufficient sedation depth and avoiding intraoperative recall. However, the EEG-derived monitor should focus on the ability to discriminate between all depths of sedation and to guide the correct hypnotic doses. Furthermore, as the depth of sedation is generally much lighter than for general anesthesia in slightly sedated patients, a reliable objective sedation entropy monitor should be able to distinguish sedation depth, rather than to guarantee a sufficient depth of sedation.

A clearly defined sedation depth facilitates the precise dose of hypnotic thereby avoiding over- or under-sedation. We have demonstrated that EEG entropy responds to the progressive deepening of propofol-sedation, and the relationship between the sedation depths is defined by both the entropy and the $\mathrm{OAA} / \mathrm{S}$ scores. The gradual reduction of RE and SE values during the progressive deepening of sedation depth was observed to correlate with the changes in the OAA/S score in agreement with previous investigations [5,15-17]. We observed a significant discrimination between light and deep sedation, which is meaningful for clinical applications compared with a previous study [17].

The original entropy scale (that continually varies between 0 and 1) was transformed to a scale of full integers between 0 and 100. A relatively large portion of the original mathematical scale of entropy values range from levels of hypnosis that can be considered too deep, whilst the most interesting range of adequate hypnosis and emergence lies between 0.5 and 1.0. For this reason, the transformation of the original continuous entropy scale is converted via a non-linear transformation (e.g., the original entropy of $0.5 / 1.0$ is transformed to a presented entropy scale of 30/100) [18]. Spectral entropy consists of both $\mathrm{RE}$ and $\mathrm{SE}$ as an immediate response to a given stimulus; RE rises first followed by an increase in SE. Vakkuri and colleagues demonstrated that RE indicates the emergence from anesthesia 11 seconds earlier than SE, and 12.4 seconds earlier than BIS; however, these were recorded evaluating both the loss and regaining of consciousness [19].

As SE in spectral entropy is devoid of EMG interference, it may prove more reliable than other EMG-sensitive, EEGderived monitors. Considering that most of the recent ICU sedation regimens do not include the use of neuromuscular blocking agents, SE may be additionally important as a reliable sedation monitor in the ICU.

When EEG-derived monitors are applied during opioidsedative combined regimens, such as propofol-remifentanil or midazolam-fentanyl, their inability to detect the opioid extra-cortical sedative effect, in addition to the high reference values in the same depth of sedation, should be considered [6]. Vanluchene and co-workers [3] observed that the sensitivity and specificity for detection of loss of responsiveness to verbal commands were decreased with increasing effect site target concentrations of remifentanil. Hernández-Gancedo et al. [4] additionally demonstrated that an overlap of entropy values corresponded to a Ramsay score of 4-6. Furthermore, recent analgesia-based sedation regimens in the ICU, rather than sedative-based sedation regimens, may require more sophisticated objective measures capable of measuring all sedation depths produced by the opioid synergistic effect of reinforcing sedation. Our investigation also demonstrates that the RE and SE values of a deeply sedated status were confined to an OAA/S of 1-2; additionally, they overlapped and were not distinguishable due to the non-linear transformation of entropy. However, RE and SE values confined to light sedation (OAA/S 3-4) were significantly different from those confined to an awake (OAA/S 5) or deep sedation (OAA/S 1-2) (Fig. 2). Considering that the usual target depth of sedation during 
the management of patients is light, these data indicate that entropy monitoring can be used, enabling the administration of hypnotic doses required to maintain adequate sedation as well as avoiding adverse outcomes from incorrect sedation levels.

Schmidt et al. [5] speculated about the limited ability of entropy measurements to distinguish between the two states of sedation (the loss of a verbal response corresponded to OAA/S score $<1$ and an awake state corresponded to OAA/S scores of $2-5)$. However, their criteria of awake did not concur with those used in the current study (OAA/S score 5). Here, the SE values confined to OAA/S scores of 5 and 4 were significantly different, whilst the RE values were indistinguishable. This indicates that SE monitoring is beneficial, probably due to the avoidance of EMG interference, particularly during the lightly sedated status. This also suggests the possible efficacy of entropy monitoring in titration sedation-depth during propofol-sedation for patients in the ICU or surgical patients with regional anesthesia who are not taking neuromuscular blockers.

The possible limitation of our study was not to evaluate its reliability in critically ill patients, in patients who asked for sedatives undergoing lower limb surgery or requiring MAC. Other confounding factors may influence entropy scores including sleep, temperature, age and drugs such as opioids, muscle relaxants, and ketamine. As the aim of the present study was to determine the entropy values specific to each depth of sedation through the OAA/S scores, the interval for each propofol concentration was not determined. Furthermore, the effect-site concentration of propofol in TCI should not guide the sedation depth alone. We observed the clinically sedative responsiveness to propofol independent of the effectsite concentration. Even at identical propofol-concentrations, patients displayed differences in the levels of sedation depth in the conventional OAA/S scale and entropy monitoring. In the current study, the depth of propofol sedation determined by both OAA/S and entropy monitoring were heavily affected by external stimuli, such as the inconvenience of determining OAA/S, rather than the propofol effect-site concentration.

In conclusion, the reduction in spectral entropy values correlated well with the reduction in the OAA/S scores during the deepening of propofol-sedation. Entropy monitoring could thus distinguish the lightly sedated status from the awake and deeply sedated status defined by the OAA/S scores. These results indicate that entropy monitoring can be utilized as a reliable objective monitor enabling the titration of a hypnotic dosage for the maintenance of adequate sedation as well as for avoiding adverse outcomes from under- or over-sedation.

\section{Acknowledgements}

The authors thank Konkuk University for providing data collection and for help with statistical analysis.

This study was supported by Konkuk University Medical Center and Konkuk University School of Medicine.

\section{References}

1. LeBlanc JM, Dasta JF, Kane-Gill SL. Role of the bispectral index in sedation monitoring in the ICU. Ann Pharmacother 2006; 40: 490500.

2. Vivien B, Di Maria S, Ouattara A, Langeron O, Coriat P, Riou B. Overestimation of bispectral index in sedated intensive care unit patients revealed by administration of muscle relaxant. Anesthesiology 2003; 99: 9-17

3. Vanluchene AL, Struys MM, Heyse BE, Mortier EP. Spectral entropy measurement of patient responsiveness during propofol and remifentanil. A comparison with the bispectral index. Br J Anaesth 2004; 93: 645-54.

4. Hernández-Gancedo C, Pestaña D, Perez-Chrzanowska H, MartinezCasanova E, Criado A. Comparing entropy and the bispectral index with the Ramsay score in sedated ICU patients. J Clin Monit Comput 2007; 21: 295-302.

5. Schmidt GN, Bischoff P, Standl T, Hellstern A, Teuber O, Schulte Esch J. Comparative evaluation of the Datex-Ohmeda S/5 entropy module and the bispectral index monitor during propofol-remifentanil anesthesia. Anesthesiology 2004; 101: 1283-90.

6. Manyam SC, Gupta DK, Johnson KB, White JL, Pace NL, Westenskow $\mathrm{DR}$, et al. When is a bispectral index of 60 too low?: Rational processed electroencephalographic targets are dependent on the sedative-opioid ratio. Anesthesiology 2007; 106: 472-83.

7. Schnider TW, Minto CF, Shafer SL, Gambus PL, Andresen C, Goodale DB, et al. The influence of age on propofol pharmacodynamics. Anesthesiology 1999; 90: 1502-16.

8. Schnider TW, Minto CF, Gambus PL, Andresen C, Goodale DB, Shafer SL, et al. The influence of method of administration and covariates on the pharmacokinetics of propofol in adult volunteers. Anesthesiology 1998; 88: 1170-82.

9. Glass PS, Bloom M, Kearse L, Rosow C, Sebel P, Manberg P. Bispectral analysis measures sedation and memory effects of propofol, midazolam, isoflurane, and alfentanil in healthy volunteers. Anesthesiology 1997; 86: 836-47.

10. Hernández-Gancedo C, Pestaña D, Peña N, Royo C, Pérez-Chrzanowska $\mathrm{H}$, Criado A. Monitoring sedation in critically ill patients: bispectral index, Ramsay and observer scales. Eur J Anaesthesiol 2006; 23 : 649-53.

11. Chernik DA, Gillings D, Laine H, Hendler J, Silver JM, Davidson $\mathrm{AB}$, et al. Validity and reliability of the Observer's Assessment of Alertness/Sedation Scale: study with intravenous midazolam. J Clin Psychopharmacol 1990; 10: 244-51.

12. Mourisse J, Lerou J, Struys M, Zwarts M, Booij L. Multi-level approach to anaesthetic effects produced by sevoflurane or propofol in humans: 2. BIS and tetanic stimulus-induced withdrawal reflex. Br J Anaesth 2007; 98: 746-55.

13. Park KS, Hur EJ, Han KW, Kil HY, Han TH. Bispectral index does not correlate with observer assessment of alertness and sedation scores during $0.5 \%$ bupivacaine epidural anesthesia with nitrous oxide 
sedation. Anesth Analg 2006; 103: 385-9.

14. Nasraway SA SA Jr, Wu EC, Kelleher RM, Yasuda CM, Donnelly AM. How reliable is the bispectral index in critically ill patients? A prospective, comparative, single-blinded observer study. Crit Care Med 2002; 30: 1483-7.

15. Anderson RE, Jakobsson JG. Entropy of EEG during anaesthetic induction: a comparative study with propofol or nitrous oxide as sole agent. Br J Anaesth 2004; 92: 167-70.

16. Lee WS, Song HC, Byun JS. Correlation between the observer's assessment of alertness/sedation and the EEG-entropy during propofol infusion for sedation under spinal anesthesia. Korean J Anesthesiol 2008; 55: 399-403.
17. Anderson RE, Barr G, Owall A, Jakobsson J. Entropy during propofol hypnosis, including an episode of wakefulness. Anaesthesia 2004; 59: 52-6.

18. Viertiö-Oja H, Maja V, Särkelä M, Talja P, Tenkanen N, TolvanenLaakso H, et al. Description of the entropy algorithm as applied in the Datex-Ohmeda S/5 entropy module. Acta Anaesthesiol Scand 2004; 48: 154-61.

19. Vakkuri A, Yli-Hankala A, Talja P, Mustola S, Tolvanen-Laakso H, Sampson T, et al. Time-frequency balanced spectral entropy as a measure of anesthetic drug effect in central nervous system during sevoflurane, propofol, and thiopental anesthesia. Acta Anaesthesiol Scand 2004; 48: 145-53. 